\title{
A Finite Difference Scheme for Time Integrations of Oscillatory Equations with Second Order Accuracy and Sharp Cut-off for High Frequencies*
}

\author{
T. Matsuno \\ Geophysical Institute, Tokyo University, Tokyo \\ (6 December 1965 and 11 January 1966)
}

\section{Introduction}

In the previous paper (Matsuno, 1966) the present author proposed a method of the numerical integrations of marching problems which works like as a high-cut filter. The particular merit of this method is that, when applied to the primitive equations, gravity waves of high frequency are suppressed, whereas largescale motions of meteorological interests remain untouched. The scheme proposed is of only first order accuracy, and has an artificial damping effect of the second power of time increment. Actual numerical computations were performed for some model patterns. The result shows that this method served as a good filter to eliminate short period gravity oscillations. However, since the damping effect is so large that one might suspect that it might raise errors of an appreciable magnitude, when this method is applied to long term integrations. In fact, Lilly (1965) discussed this point by carrying out numerical integrations and concluded that this method was much less accurate compared with other methods which have at least second order accuracy.

However the author considers that his results should not be directly applied to the estimations of errors of actual numerical integrations of meteorological problems, because under usual conditions the ratio of time increment to the period of meteorological waves is of the order of $10^{-2}$, while in the case of Lilly's computation this ratio was some $10^{-1}$ or more. In the former situation

\footnotetext{
* Division of Meteorology, Contribution. No. 145.
}

the artificial damping effect could be ignored against the physical damping due to viscosity. At any rate the scheme proposed by the author was directed to the elimination of errorneous short period oscillations and it was inferior to other methods in the accuracy. On the contrary, all those methods which were recommended by Lilly as stable and accurate methods have amplification factor larger than unity and larger for higher frequencies. Of course, it is possible to keep the total amplification in a given integration period within some limit by reducing time increment to sufficiently small value. But such methods are not practical for integrations of the primitive equations. The system treated has a large spectrum range, and we are interested in only the lowest frequency part of the spectrum. In order to suppress unrealistic growth of incipient high frequency oscillations we need to take very small value as a time increment. Therefore it is desirable to get a time integration scheme which has the accuracy at least up to the second power of time increment and at the same time has amplification rate smaller than unity for very high frequencies. The purpose of this short note is to present a numerical integration scheme which is an extension of author's previous methods, and which gives a better accuracy keeping the damping effects for very high frequencies.

Kurihara (1965) examined various time integration schemes of implict and iterative type and discussed their filtering characteristics. Among them the leap-frog trapezoidal method (according to his nomenclature) has the second order accuracy and damping effect 
for high frequency oscillations. But it differs from the method described below in the two points. First the latter is a single time level method and it is therefore free from the socalled computational mode and second the damping effect of the latter method is much larger than the former for very high frequency oscillations while for low frequency part the damping effects of the two are of comparable magnitude.

\section{Description of the methods and the nu- merical test}

We shall confine ourselves only to single time level method, i.e. the values of dependent variables at $(n+1)$-th time step must be determined by those at $n$-th step. Then the amplification constant, when it is applied to an oscillation equation, turns to a power series of $(i \omega)$, where $\omega$ is the frequency measured by time increment as the unit. Since we are requiring the second order accuracy, the amplification constant $\lambda$ has to be

$$
\lambda=1+i \omega-\frac{1}{2} \omega^{2}-\frac{\alpha}{2} i \omega^{3}
$$

where $\alpha$ is a numeric factor to be determined so as to get the best filtering characteristics for our purpose.

The scheme which should have the eigenvalue $\lambda$ expressed as (2) will be constructed in the following manner. We shall consider the following equation

$$
\frac{d \xi}{d t}=\Omega \xi
$$

together with the relation

$$
\Omega \xi=i \omega \xi
$$

where $\Omega$ is a linear operator on $\xi$ and $i \omega$ is an eigenvalue of $\Omega$.

Since any power of $\Omega$ and linear combinations of them have the same eigenvector $\xi$, the operator $L$ which has the eigenvalue given as (2) is written as

$$
\begin{aligned}
L & =I+\Omega+\frac{1}{2} \Omega^{2}+\frac{\alpha}{2} \Omega^{3} \\
& =I+\Omega\left(I+\frac{1}{2} \Omega(I+\alpha \Omega)\right)
\end{aligned}
$$

where I stands for the unit operator.

The procedure expressed as (6) may be written as following, when it is applied to the equation $d x / d t=f(x)$,

$$
\begin{aligned}
& x_{*}^{(n+\alpha)}=x^{(n)}+f\left(x^{(n)}\right) \cdot \alpha \Delta t \\
& x_{*}^{\left(n+\frac{1}{2}\right)}=x^{(n)}+f\left(x_{*}^{(n+\alpha)}\right) \frac{1}{2} \Delta t \\
& x^{(n+1)}=x^{(n)}+f\left(x_{*}^{\left(n+\frac{1}{2}\right)}\right) \Delta t
\end{aligned}
$$

where asterisked quantities appear only as intermediate ones.

The filtering characteristics change conti-

\begin{tabular}{|c|c|c|c|c|}
\hline Ref. No. & $\alpha$ & $\lambda$ & $\begin{array}{l}\text { Stability Condition } \\
\qquad|\lambda|<1\end{array}$ & Remarks \\
\hline 1 & 0 & $\left(1+\frac{1}{4} \omega^{4}\right)^{\frac{1}{2}}$ & - & \\
\hline 2 & $1 / 4$ & $\left(1+\frac{1}{64} \omega^{6}\right)^{\frac{1}{2}}$ & - & $\begin{array}{l}|\lambda| \text { is the most close to } 1 \text {, } \\
\text { slightly amplifying }\end{array}$ \\
\hline 3 & $1 / 3$ & $\left(1-\frac{1}{12} \omega^{4}+\frac{1}{36} \omega^{6}\right)^{\frac{1}{2}}$ & $\omega<\sqrt{3}$ & $\begin{array}{l}\text { Accurate up to the third order } \\
\lambda=1+i \omega+\frac{1}{2 !}(i \omega)^{2}+\frac{1}{3 !}(i \omega)^{3}\end{array}$ \\
\hline 4 & $1 / 2$ & $\left(1-\frac{1}{4} \omega^{4}+\frac{1}{16} \omega \omega^{6}\right)^{\frac{1}{2}}$ & $\omega<2$ & Stable region is the largest \\
\hline 5 & 1 & $\left(1-\frac{3}{4} \omega^{4}+\frac{1}{4} \omega^{6}\right)^{\frac{1}{2}}$ & $\omega<\sqrt{3}$ & $\begin{array}{l}\text { Filtering effect is the strongest } \\
\qquad|\lambda(\omega=\sqrt{2})|=0\end{array}$ \\
\hline
\end{tabular}
nuously with the change of $\alpha$. In Table 1, the absolute values of the amplification factor and some other properties are listed for some particular values of $\alpha$.

In Fig. 1 the absolute magnitude of $\lambda$ are shown as functions of $\omega$ for the five cases

Table 1. Filtering characteristics of the scheme given by (6) for some values of 


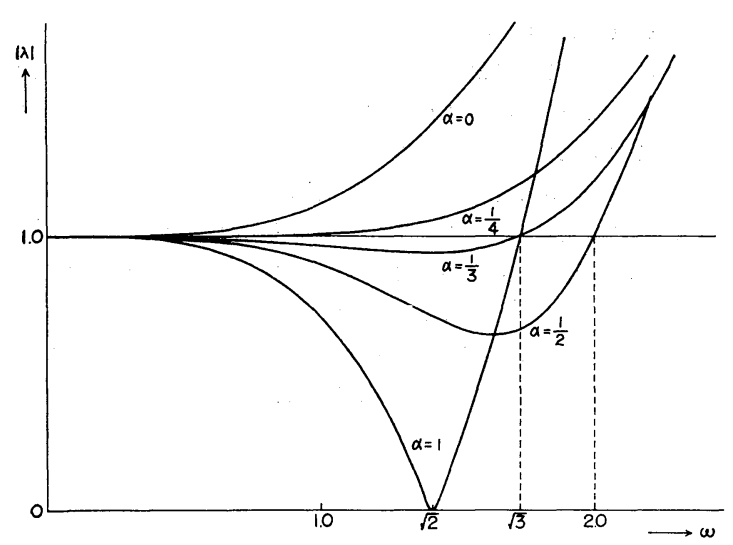

Fig. 1. The absolute values of amplification factors for various values of parameter $\alpha$.

listed above.

The method 1 is the two step iteration method which gives the best accuracy (the second order in $\Delta t$ ). This method has amplifying effect for any oscillations.

The method 2 gives the higher accuracy than any other methods so far as the absolute value of amplification rate for pure oscillations are concerned. Namely if the method 2 is used for integrating equations of oscillatory type, the amplitudes of neutral oscillations will be kept almost unchanged.

The method $3(\alpha=1 / 3)$ has the third order accuracy, i.e., the truncation error is of the fourth power of $\Delta t$, when the dependent variables are expanded in power series. Therefore the method 3 is identical with the RungeKutta method of the third order accuracy. It is interesting that this method has a damping effect when it is applied to an oscillatory equation.

It should be remarked that in these schemes the best accurate method (method 3 ) is different from the method which keeps the amplitude of a neutral oscillation most close to unity (method 2). The merit of the method 4 is that it gives the largest stable region. Namely the absolute value of amplification constant is smaller than unity for $\omega<2$. The two step iteration method that has this property is the one proposed by the present author in the previous paper. The procedures of the method 4 are interpreted in the following manner: At first the values of dependent variables at $1 / 4 \Delta t$ are calculated by use of the foreward difference method. Then we can get the values at $1 / 2 \Delta t$ by use of the centered difference method, and finally we get the values at $\Delta t$. The method corresponding to this procedure in the case of two step iteration is the one that is refered as method 1 in this paper.

The method 5 is characterized by a very strong damping effect for high frequency oscillations. The amplification rate becomes null at $\omega=\sqrt{2}$. Since the absolute value of $\lambda$ deviates from unity in the forth power of $\Delta t$ near $\omega=0$, the filtering characteristics of this method shows a very sharp cut-off for the high frequency part.

The numerical integrations by use of these schemes were performed for the examples given by Lilly (1965).

The equations treated were simultaneous first order differential equations for four dependent variables. They make a non-linear oscillatory system and show different behaviours depending upon the initial conditions. This system is a particuler form of the simplified vorticity equation and conserves two quadratures, one of which is the kinetic energy. According to the Lilly's paper, three sets of initial conditions are adopted as following ;

Case A: The four variables approach asymptotically certain constants.

Table 2. Ranges of the variations of the energy for the integration period by various methods

\begin{tabular}{c|c|c|c}
\hline & Case A & Case B & Case C \\
\hline Method 2 & $1.0000-0.9985$ & $1.0000-0.9986$ & $1.0000-0.9997$ \\
\hline Method 3 & $1.0000-0.9985$ & $1.0000-0.9973$ & $1.0000-0.9980$ \\
\hline Method 4 & $1.0000-0.9985$ & $1.0000-0.9932$ & $1.0000-0.9939$ \\
\hline Method 5 & $1.0022-0.9941$ & $1.0021-0.9807$ & $1.0002-0.9823$ \\
\hline
\end{tabular}


Case B: Small perturbations are added to the case A.

Case C: The four variable share the energy equally.

In Table 2, the ranges of the variation of the energy in the integration period $(0 \sim 100$ non-dimensional time) are shown.

The results of the numerical integration tests make sure the analyses made earlier, assuming that the method is applied to a linear oscillation equation.

By using method 2 the energy fluctuated in the range shown in the table, but no secular trend was found in this integration period. By using the method 5 (the strongest filter) the energy decreased most markedly. In Fig. 2 the change of the energy by use of the method 5 is shown (curve C) overlapped on the figure taken from the Lilly's paper.

Even by use of this method the energy loss

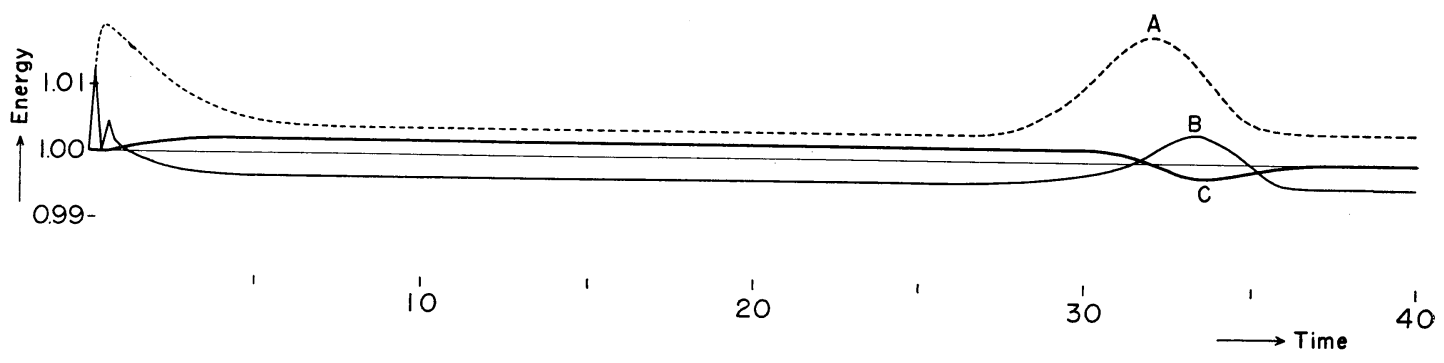

Fig. 2. Comparison of the accuracy of the present method with those of other methods. Plotted on the figure after Lilly (1965).

A: Adams-Bashforth method.

B: Miyakoda's multi-time level method.

C: Present Method.

Refer to Lilly's paper for the former two.

due to the artificial damping effect is compared to those in the case of using the other method that are described in the Lilly's paper as accurate ones.

So far as we observe the table 2 the method 5 is the worst of the four scheme presented there. However if we treat the primitive hydrodynamic equations, we have to suppress errorneous short period oscillations. In those occasions, the stronger the damping effect of the scheme is, the more stably the numerical integration would be performed.

In conclusion the author would like to stress that a suitable computation scheme should be selected according to the natures of problems to be treated. Since the proposed method has some particular characters which no other methods have, it might be applied for the problems which need them.

\section{Acknowledgments}

The author thanks to Prof. S. Syonno for stimulating the author to do this work.

\section{References}

Kurihara, Y., 1965: On the use of implicit and interative methods for the time integration of the wave equation. Mon. Wea. Rev. 93, 33-46.

Lilly, D.K., 1965: On the computational stability of numerical solutions of time-dependent nonlinear geophysical fluid dynamics problems. Mon. Wea. Rev. 93, 11-26.

Matsuno, T., 1966: Numerical integrations of primitive equations by use of a simulated backward difference method. J. meteor. Soc. Japan, 44, 1. 76-84.

\title{
二次の精度をもちかつ高周波に対して強い減衰作用をもつ時間皘分方式
}

\author{
松野 太 郎
}

